\title{
Influence of Stochastic Mesoscopic Structure on Macroscopic Mechanical Behavior of Brittle Material
}

\author{
Y.F. Fu' ${ }^{1}$, M.L. Huang ${ }^{2}$ and C.A. Tang ${ }^{1,3}$ \\ ${ }^{1}$ CRISR, Resources and Civil Engineering School, \\ Northeastern University, Shenyang 110006, China P.R. \\ ${ }^{2}$ Resources and Environment School, Liaoning University of Engineering \\ and Technology, Fuxin 123000, China P.R. \\ ${ }^{3}$ LNM, Institute of Mechanics, Chinese Academy of Science, \\ Beijing 100080, China P.R.
}

Keywords: Failure, Heterogeneity, Localization, Mesoscopic, Numerical Simulation

\begin{abstract}
A newly developed numerical code, MFPA ${ }^{2 \mathrm{D}}$ (Material Failure Process Analysis), is applied to study the influence of stochastic mesoscopic structure on macroscopic mechanical behavior of rock-like materials. A set of uniaxial compression tests has been numerically studied with numerical specimens containing pre-existing crack-like flaw. The numerical results reveal the influence of random mesoscopic structure on failure process of brittle material, which indicates that the variation of failure mode is strongly sensitive to the local disorder feature of the specimen. And the patterns of the crack evolution in the specimens are very different from each other due to the random mesoscopic structure in material. The results give a good explanation for various kinds of fracture modes and peak strength variation observed in laboratory studies with specimens made from the same rock block being statistically homogenous in macro scale. In addition, the evolution of crack is more complicated in heterogeneous cases than in homogeneous cases.
\end{abstract}

\section{INTRODUCTION}

Essentially, the failure and deformation of rock-like materials is a process of progressive evolution in which the cracks initiate, propagate, interact and coalesce due to external loading. The results from the observations of laboratory and field show that the macroscopic mechanical behavior of highly heterogeneous rock-like material is pronouncedly influenced by the mesoscopic heterogeneity. With regard to failure, many beneficial investigations [1-3] were carried out in the past decades in both experiments and the theory, but most of studies on the mechanism of rock failure are conducted just based on the assumption that rock is homogeneous medium, the influence of the heterogeneity on failure process is seldom considered. However, many rock failure phenomena, such as the activity of induced seismicity, the echelon pattern fracture in crust deformation, the precursor, aftershock of source development of earthquake, etc., are closely related to the heterogeneity of rocks. Hence, the heterogeneity of material must be considered in studying the behavior of rock-like materials. However, there is lacking of mature theory to describe the heterogeneity, the basic character of rock. Nowadays the numerical simulation based on statistical mesoscopic damage 
mechanics is regarded as an effective tool to resolve the problem. Of later years, a code of MFPA ${ }^{2 \mathrm{D}}$ (Material Failure Process Analysis), based on the principles of FEM is developed at Center for Rockburst and Induced Seismicity Research, Northeastern University. And Tang et al [4,5] has studied many problems about failure mechanisms of rock-like materials with MFPA ${ }^{2 \mathrm{D}}$. In this paper, by conducting a set of numerical tests on specimens containing pre-existing crack-like flaw under uniaxial compression, MFPA $^{2 D}$ is applied to study the influence of stochastic mesoscopic structure on macroscopic mechanical behavior of rock-like materials.

\section{OUTLINE OF MFPA ${ }^{2 D}$ AND MODEL SETUP}

\subsection{Brief description of $\mathrm{MFPA}^{2 \mathrm{D}}$}

The MFPA code, developed at the Center for Rockburst and Induced Seismicity Research (CRISR), Northeastern University, China, can be used for modeling the evolution of damage or crack initiation, propagation and coalescence in brittle material. The mesoscopic heterogeneity of material properties can be considered in the code, based on FEM. The mesoscopic heterogeneity represents the variation of the mechanical properties for the elements composed of the material. The consideration of heterogeneity for the elements composed of the material is achieved to assign to the elements random strength and elastic modulus by assuming a Weibull's distribution. The distribution is defined by equation (1) with three parameters, $a, a_{0}$ and $m$.

$$
\varphi(\alpha, m)=m \cdot\left(\frac{\alpha}{\alpha_{0}}\right)^{m-1} \cdot e^{-\left(\frac{\alpha}{\alpha_{11}}\right)^{m}}
$$

where the $a$ is the element strength or elastic modulus and $a_{0}$ is the mean strength or elastic modulus of elements for the material. The parameter $m$, defined as homogeneity index of the brittle material, controls the shape of the distribution function, which relates to the degree of material heterogeneity. Materials with higher $m$ values represent more homogeneous materials, whereas those with lower $m$ values, more heterogeneous materials.

The more detailed explanation about the $\mathrm{MFPA}^{2 \mathrm{D}}$ can be found in the literature [6].

\subsection{Model setup}

The specimens with different $m$ value are numerically tested:

1. Four specimens with value $m=2.0$ and same mechanical properties of elements, to study the influence of stochastic mesoscopic structure on the macroscopic behavior,

2. Three specimens with different value $m=1.1,2.0,5.0$, to investigate the effect of heterogeneity.

In all cases, the specimen undergoes plane stress compression. The geometrical and mechanical properties for all the specimens are listed in Table 1 . The schematic map ( $a, b$ and $c$ ) and statistical distribution of stréngth for elements of specimens $\left(a^{\prime}, b^{\prime}\right.$ and $\left.c^{\prime}\right)$ are in Fig.1.

Table 1. Mechanical and geometrical parameters for the specimens

\begin{tabular}{cc}
\hline Parameter & Value \\
\hline Homogeneity index $(m)$ & $1.1 .2 .0,5.0$ \\
Mean compressive strength $\left(\sigma_{0}\right)$ & $400 \mathrm{MPa}$ \\
Mean elastic Modulus $\left(E_{0}\right)$ & $60000 \mathrm{MPa}$ \\
Tension cutoff $(\lambda)$ & 0.1 \\
Frictional angle $(\phi)$ & $30^{\circ}$ \\
\hline
\end{tabular}




\begin{tabular}{cc}
\hline Size $(\mathrm{mm})$ & $100 \times 50$ \\
Mesh & $200 \times 100$ \\
\hline
\end{tabular}

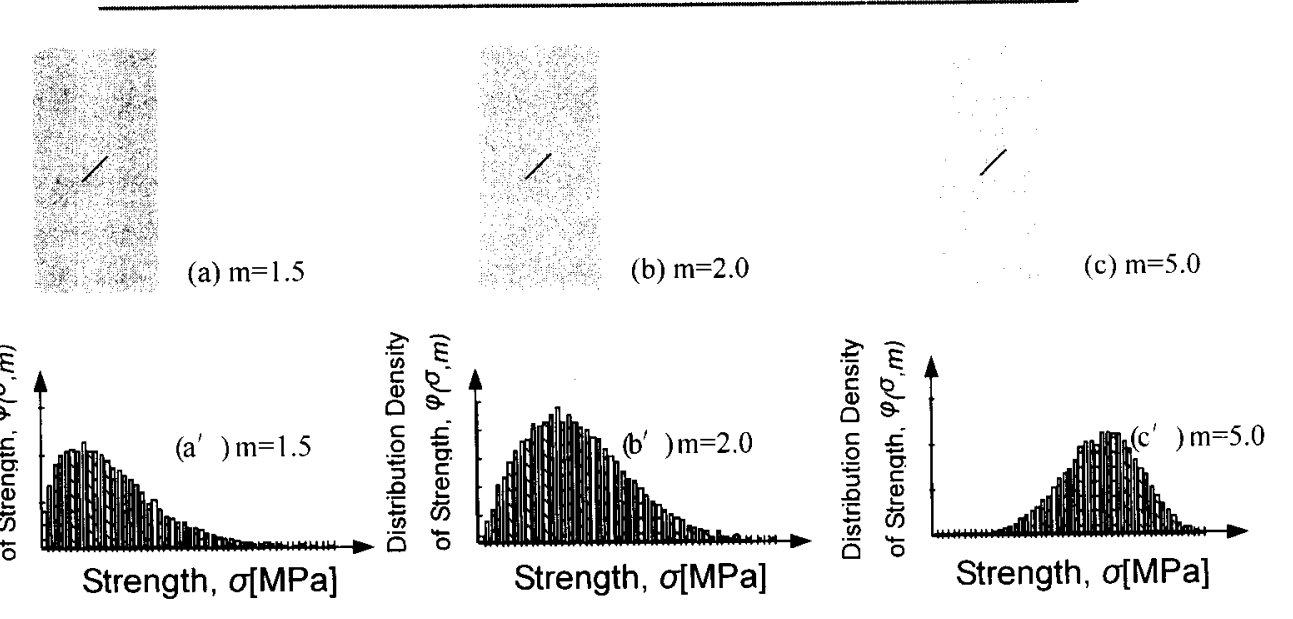

Fig. 1. Schematic map of specimens

\section{NUMERICAL SIMULATION RESULTS AND DISCUSSION}

\subsection{Complete stress-strain curve and stress distribution and redistribution}

In the case of studying crack propagation in rock specimen, the assumption regarding rock as homogeneous and continuous material can make the mathematical process more convenient, but the

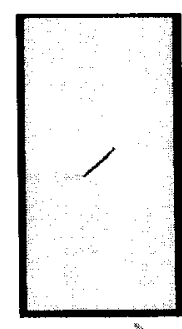

Step $=10$
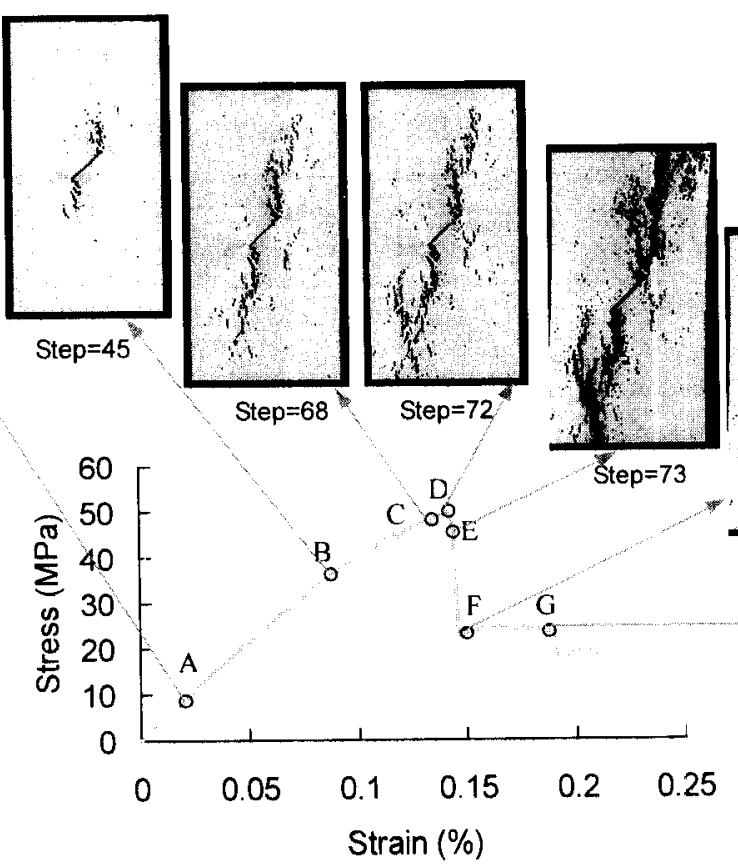

Fig. 2 Failure process of specimen No.1 
results caused by the assumption maybe are unaccepted.

Fig. 2 shows the failure process of specimen No.1 with homogeneity index $m=2.0$. Although the pattern of crack propagation is very complicated, it seems closer to the reality of rock failure than the prediction from fracture mechanics based on the assumption of homogeneity continuity. The whole process can be divided into three stages: linear deformation, non-linear deformation and softening, as the following:

1. The stage of linear deformation. During the stage of initial loading the concentrated stress in tips of the pre-existing flaw doesn't reach the critical strength of elements, so the crack propagation doesn't occur. In this time, the macroscopic stress field is still statistically coincided with the analytic solution of fracture mechanics because few elements consisting of the specimen fail during this stage (as shown in step 10). Consequently, the stress-strain curve shows good linearity.

2. The stage of non-linear deformation. With increasing loading, the crack initiates at the tip of the flaw. Although the overall direction of the crack propagation is more or less the same as predicted by fracture mechanics theory, the formation of crack propagation is rather complex and shows discontinuous features. The boundary of crack is very vague and the crack bifurcation occurs frequently (as shown in step 45,68, and 72). The distribution of stress field gradually changes owning to the increasing number of failure elements and random position of their emergence. The stressstrain curve demonstrates non-linearity.

3. The softening stage. Although the stress decreases 9 percent from the point $D$ to the point $E$, the climax of number of failure elements occurs (as shown in step 73). After the peak-strength the crack has almost crossed the section of the specimen but still showed a non-zero residual stress (as shown in step 75,95 ) and further loading is difficult to drive the cracks for a visible propagation.

\subsection{The influence of stochastic mesoscopic structure on macroscopic mechanical behavior}

There is a well-known fact that even core specimens obtained from a seemingly homogeneous block of rock show variability both in deformation and strength properties due to the stochastic mesoscopic structure in a specimen. It means that the randomly distributed micro-defects in the specimen result in localization of deformation and failure in different position. In order to study the problem, four specimens with same macroscopic mechanical properties but with different mesoscopic structure are numerically tested subjected to compressive loading. In Fig.3, the numerically obtained stress-strain curves of four specimens are shown. For the whole deformation process of the curves, linear, non-linear, unstable and residual deformation, four stages are suffered for every one of the specimens. All curves are coincided very well during linear and partly non-linear stage before reaching peak strength, and it demonstrates that during the initial stage of low damage the spatial structure of materials is not strongly destroyed, but has weak statistical

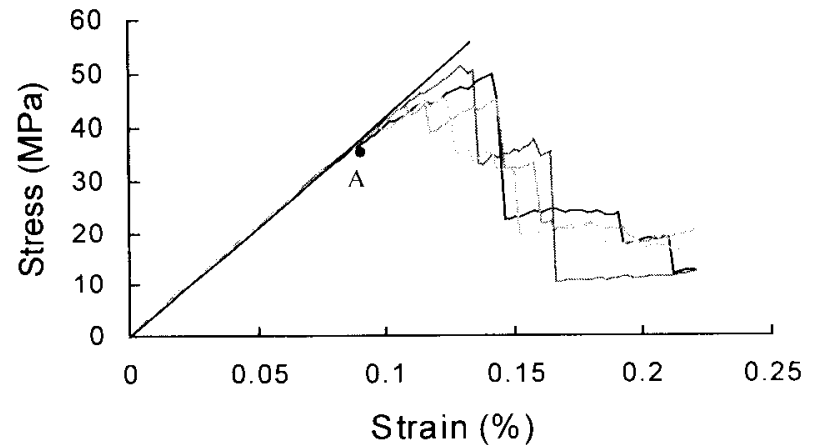

Fig. 3 Stress-strain curves for the four specimens with $\mathrm{m}=2.0$ fluctuation influencing the macroscopic behavior slightly. However, after beyond the point $A$, the 
situation is completely changed. Because of development of micro-fracture in specimen, the original distribution of mechanical properties for elements is changed to larger extent. The macroscopic behaviors of four specimens show pronounced difference in that the curves demonstrate obvious specimen-specific behavior. Although the peak strengths of all specimens vary in a small variation, during the post-peak strength the softening formation of four specimens show larger discretion. On one hand, the softening process is very fast and sudden stress drop occurs for some of them. On the other hand. the weakening process is smooth and progressive failure behavior is observed for other specimens. During the portion of residual strength, the softening for any of the specimens tends to reach a certain value.

In a word, during the stages of initial loading and residual strength portion the macroscopic mechanical behavior is not sensitive to the distribution of mechanical properties for elements, but the situation is completely different during stages of macroscopic fracture of the specimen and softening process. The numerical simulations show that the softening process in the post-peak portion is structurally unstable in which a small difference in mesoscopic structures of the specimens can be strongly enlarged at certain point that leads to big difference in patterns of softening behavior. The results from the simulations agree well with the results obtained by Xia [7].

Fig. 4 reveals the fracture patterns of four specimens. The patterns can be divided into two types. The first is the cleavage alone the wing crack of the top and bottom tip of the pre-existing flaw. The second is mixed cleavage including tensile and shear cracks. The first type of fracture demonstrates more strong brittleness than the second type because tensile fracture releases less energy than shear. For the four specimens, the macroscopic mechanical properties, geometry and size of the preexisting flaw are completely the same, but

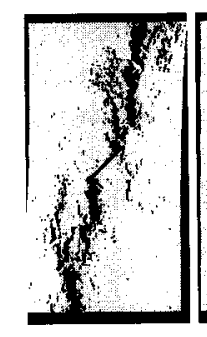

Fig. 4 Fracture pattern of rock specimen with pre-existed flow their fracture patterns show statistical nature and hardly have identical form. In comparison with observations from laboratory, it can be concluded that the distributive character of the heterogeneity plays a crucial role in determining the evolution of fracture, and is a main and rigueur aspect about rock failure. In theory it explains the essential reason of variation and randomicity of failure phenomenon in rock-like materials.

\subsection{The influence of homogeneity index $m$ on macroscopic mechanical behavior}

Fig.5 shows fracture pattern of specimens with different homogeneity index 1.5, 2.0 and 5.0. With increasing homogeneity index $m$, the pattern of crack propagation tends to the predicted pattern from fracture mechanics theory, that is, the crack is more smooth and continuous, and the micro-fractures almost concentrate on the advanced tip of the pre-existing flaw or along the path of wing crack propagation. It seems to us that the analytical solution in fracture mechanics is not effective to be used to describe the problems in which the heterogeneity has to be considered. Fig. 6 also reveals the same role

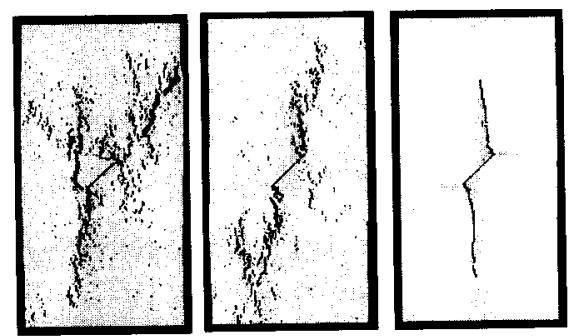

Fig. 5 Fracture mode and of specimens with Different homogeneity index $\mathrm{m}=1.5,2.0,5.0$ 
that the linearity and the brittleness are obviously enhanced with increasing the homogeneity index $m$. Because increasing the heterogeneity of specimen raises the number of low strength element (as shown in Fig.1a', b', c') and the variation magnitude of the local stress concentration that results in macrofractures in a lower stress level, at last it leads to the strength reduction of specimens.

\section{CONCLUSION}

The following conclusions are obtained from the numerical simulations:

1. The influence of stochastic mesoscopic structure on fracture

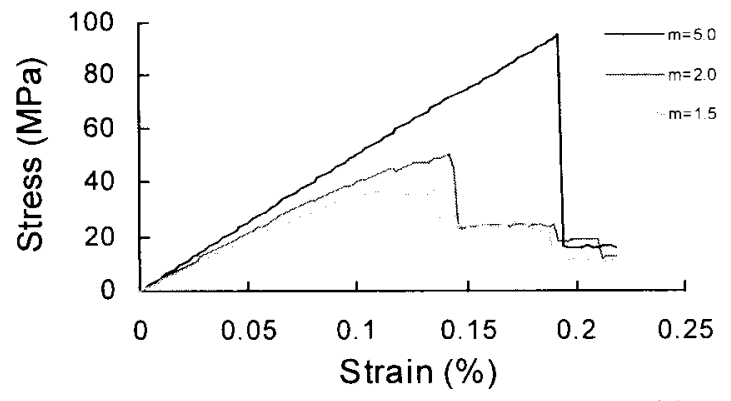

Fig. 6 Stress-strain curve of specimens with different homogeneity index $\mathrm{m}=1.5,2.0,5.0$ mode is much higher than on the peak-strength variation. The macroscopic mechanical behavior is more sensitive to mesoscopic structure after peak strength than before peakstrength, and shows strong specimen-specific behavior. The macroscopic failure of specimens demonstrates catastrophic character and shows different fracture modes.

2. With increasing homogeneity index $m$ the macroscopic strength of specimen rises, macroscopic linearity is more obvious, and the macroscopic failure process presents markedly brittle behavior. At the same time, the pattern of crack propagation is closer to the pattern predicated from fracture mechanics, that is, the path of crack propagation is more continuous and smooth.

AKNOWLEDGEMENT: The work presented in this paper was supported by the Chinese National Key Fundamental Research "973 Programme" (No.95-13-07-01) and the National Natural Science Foundation (No.49974009).

\section{REFERENCE}

1. N.G.W.Cook, The failure of rock, Int. J. Rock Mech. Min. Sci., 2 (1965) p.389.

2. R. Chen, H. Yan, Deformation precursor of rock failure in Laboratory, ACTA GEOPHYSICA SISICA, 32 Supp.1 (1989) p.246.

3. Tao Zhenyu, Notes on recent development of rock mechanics, ADVANCES IN MECHANICS, 22(2) (1992) p.161.

4. C.A. Tang, A New Approach to Numerical Method of Modeling Geological Processes and Rock Engineering Problems, Engineering Geology, 49 (1998) p.207.

5. C.A. Tang and P.K. Kaiser, Numerical Simulation of Cumulative Damage and Seismic Energy Release during Brittle Rock Failure-Part I. Fundamentals. Int. J. Rock Mech. \& Min. Sci. 35(2) (1998) p.113.

6. C. A. Tang, Numerical simulation of progressive rock failure and associated seismicity. Int. J. Rock Mech. Min. Sci., 34(2) (1997) p.249.

7. M. F. Xia, W. S. Han, F. J. Ke and Y. L. Bai, Statistical mesoscopic damage mechanics and damage evolution induced catastrophe (II), ADVANCES IN MECHANICS, 25(1) (1995) p.1. 


\section{Fracture and Strength of Solids IV}

10.4028/www.scientific.net/KEM.183-187

Influence of Stochastic Mesoscopic Structure on Macroscopic Mechanical Behavior of Brittle Material 10.4028/www.scientific.net/KEM.183-187.785

\section{DOI References}

[4] C.A. Tang, A New Approach to Numerical Method of Modeling Geological Processes and Rock Engineering Problems, Engineering Geology, 49 (1998) p.207. doi:10.1016/S0013-7952(97)00051-3

[5] C.A. Tang and P.K. Kaiser, Numerical Simulation of Cumulative Damage and Seismic Energy Release during Brittle Rock Failure-Part I. Fundamentals. mt. J. Rock Mech. \& Mi Sci. 35(2) (1998) p.113. doi:10.1016/S0148-9062(97)00009-0 